\title{
Uber Schmelzvorgang und Wärmehaushalt im Zentralgebiet des Inlandeises
}

\author{
Von Paul Jaspersen in Kiel
}

$\mathrm{Zusammenf}$ assung. In der folgenden Arbeit wird der Nachweis geführt, daß auch in größerer Tiefe der zentralen grönländischen Eiskappe, abgesehen von der Bodenzone, ein Schmelzen nicht stattfindet.

A bstract. In the following paper evidence is produced that also in great depth of the central Greenland ice-cover no melting can take place, setting aside the zone on the bottom.

Nachdem P. WoLDSTEDT (1952) die Bewegungsvorgänge im Inlandeis in klaren Gedanken geschildert hat, erscheint es wünschenswert, auch die Frage des Schmelzens im Innern des Inlandeises und des Wärmehaushalts im zentralen Bereich zu erörtern. P. Woldstedt und andere Forscher nehmen an, daß bei dem Absinken der Schnee.* und Eisschichten von der Oberfläche bis zur Sohle des Inlandeises in einer gewissen, aber unbekannten Tiefe der Schmelzpunkt des Eises erreicht werde; dieser liegt um so weiter unter $0^{\circ} \mathrm{C}$, je stärker der Druck ist. Ob aber wirklich der Schmelzpunkt erreicht wird und dann auch tatsächlich das Eis zum Schmelzen kommt, hängt davon ab,

1. ob zunächst das Eis auf die Temperatur des Schmelzpunktes erwärmt wird,

2. ob dann noch die für das Schmelzen selbst erforderliche Wärmemenge vorhanden ist.

Zur Veranschaulichung der Verhältnisse und zur Erkennung der verschiedenen möglich erscheinenden Vorgänge soll das Beispiel des grönländischen Inlandeises zunächst in etwas schematisierter Form behandelt werden. Zur rechnerischen Erfassung möge hierbei eine Säule des Inlandeises unter der Eismitte von $1 \mathrm{qm}$ Querschnitt und einer Höhe etwa gleich der Eisdicke gewählt werden.

Die „Französische Polarexpedition in Grönland und Adelieland (Antarktis) 1948-51“, gibt auf Grund neuerer seismischer Messungen diese Eisdicke mit $3100 \mathrm{~m}$ an, wobei die Oberfläche des Untergrunds etwa im Meeresniveau festgestellt wurde (KanNENBERG 1951). Um dem verschiedenen spezifischen Gewicht der Schnee-, Firn- und Eisschichten angenähert Rechnung zu tragen, möge zur Vereinfachung der Gewichtsberechnung eine Säule kompakten Gletschereises von $2500 \mathrm{~m}$ im Weiteren zugrunde gelegt werden. Unter der Voraussetzung, daß das Inlandeis sich im dynamischen Gleichgewichtszustand zwischen Zuwachs und Abfluß befinde, wird der jährliche Zuwachs durch Niederschläge durch das Absinken der ganzen Säule um das gleiche Maß ausgeglichen. Die Niederschlagshöhe auf der Eismitte kann mit etwa $34 \mathrm{~cm}$ (auf Wasser reduziert) oder $340 \mathrm{~kg}$ je qm angenommen werden (K. Wegener, Bd. III, S. 117). Dieser Wassersäule entspricht eine Eissäule von $38 \mathrm{~cm}$ bei einem spezifischen Gewicht von 0,9 (BRINkmanN I, S. 33). Um diesen Betrag wird also auch jährlich die angenommene Eissäule absinken.

Die maximale Herabsetzung des Schmelzpunktes muß sich am Boden ergeben. Das Gewicht der Eissäule beträgt bei einem spezifischen Gewicht von 0,9 etwa $250000 \mathrm{~kg}$, woraus sich am Boden ein Druck von 225 atm. ergibt. Da nun der Schmelzpunkt durch Druck um $0,008^{\circ} \mathrm{C}$ für je $1 \mathrm{~atm}$. herabgesetzt wird (ebenda S. 34), errechnet sich die Temperatur des Schmelzpunktes an der Eissohle auf rund $-2^{\circ} \mathrm{C}$.

Di e Größe des Wärmebedarfs, wenn am Boden der Eissäule von $2500 \mathrm{~m}$ die Temperatur des Schmelzpunktes in der gesamten jährlich am Grund eintreffenden Eismenge von $340 \mathrm{~kg}$ erreicht werden soll, ergibt sich aus Folgendem: Die Temperatur des Firns nahe der Oberfläche beträgt auf Eismitte etwa $-28^{\circ} \mathrm{C}$ (K. WEGENER, III, 
S. 204 ff.). Die Erwärmung bis zum Schmelzpunkt am Boden müßte also $26^{\circ} \mathrm{C}$ betragen. Das Eis sinkt in Jahrtausenden von der Oberfläche bis zum Grunde; aber in jedem Jahr müßte eine Wärmemenge aufgebracht werden, die gleich wäre der Wärmemenge, die erforderlich ist, um die jährliche Niederschlagsmenge von $340 \mathrm{~kg}$ um $26^{\circ} \mathrm{C} \mathrm{zu} \mathrm{er-}$ wärmen. $\mathrm{Da}$ die spezifische Wärme des Eises 0,5 ist, sind dazu $340 \cdot 26 \cdot 0,5 \mathrm{WE}=$ 4420 WE notwendig. Darüber hinaus müßte für das Schmelzen selbst die latente Wärme von $80 \mathrm{WE}$ je $1 \mathrm{~kg}$ Eis aufgebracht werden.

Welche Wärmemenge steht dagegenzur Verfügung? Durch die von der Oberfläche bis zur Sohle absinkenden Eisschichten wird eine mechanische Arbeit geleistet, die sich nach Maßgabe des mechanischen Wärmeäquivalents, $427 \mathrm{mkg}=1 \mathrm{WE}$, in Wärme umsetzt. Beim Absinken der Eissäule von $2250000 \mathrm{~kg}$ um $0,38 \mathrm{~m}$ entsteht jährlich eine Arbeitsleistung von $855000 \mathrm{mkg}$, die einer Wärmemenge von rund $2000 \mathrm{WE}$ entspricht. Andere Wärmequellen sind im Zentralgebiet nicht gegeben. Die Wirkung der Sonnenstrahlung ist in der oben genannten Temperatur des Firns nahe, aber unter der Oberfläche, schon eingeschlossen (K. WEgener, III, S. 48 Fußnote). Von der Reibungswärme, die das Eis auf seinem Wege nach unten teils im Innern, teils am Grunde erzeugt, geht kaum etwas verloren, da der erstere Teil im Eis verbleibt und unmittelbar der Erwärmung des Eises dient, während der letztere dem aus der Erde kommenden Wärmestrom zugeführt wird. Es stehen aber einem Wärmebedarf von $4420 \mathrm{WE}$, der nur zur Erwärmung des Eises bis zum Schmelzpunkt dienen würde, höchstens 2000 WE verfügbar gegenüber und auch diese nur zum Teil, weil die Reibungswärme teilweise erst am Grunde entsteht.

Daraus ist zunächst der Schluß zu ziehen, daß eine allmähliche Vorerwärmung der Eissäule bis auf den Schmelzpunkt auch dann nicht eintreten könnte, wenn, als Grenzfall betrachtet, die gesamte erzeugte Wärmemenge schon auf dem Wege des Eises oberhalb der Sohle hierfür zur Verfügung stände; erst recht fehlen die Kalorien, die als latente Wärme zum Schmelzen des Eises weiter erforderlich wären. Die ganze Eissäule wird also bis zur Sohlenzone im festen Aggregatzustand verbleiben, wenn auch infolge des hohen Druckes mit stark plastischer Eigenschaft.

Wie sich die Gesamterzeugung an Wärme auf das Eisinnere und die Sohlenzone verteilt, ist nicht festzustellen. Die obige Rechnung erfaßt den theoretischen Grenzfall, daß die gesamte potentielle Energie schon im Innern des Eises in Reibungswärme umgesetzt würde. Wenn jetzt der entgegengesetzte Grenzfall angenommen würde, daß die gesamte Wärme erst am Grunde erzeugt würde, so würde das bedeuten, daß das Eis mit unveränderter Oberflächentemperatur unten einträfe. In diesem ebenfalls nur theoretischen Fall würde die verfügbare Wärme aber nicht auf die große Eismenge der ganzen Säule verteilt, wobei nach obiger Untersuchung kein Schmelzen erzielt würde, sondern konzentriert in beschränktem Raum wirksam werden. Hierbei würde die Wärme zum Schmelzen einer gewissen Eismenge ausreichen. Je $1 \mathrm{~kg}$ Eis würden zunächst $1 \cdot 26 \cdot 0,5 \mathrm{WE}=13 \mathrm{WE}$ zur Vorerwärmung und weitere $80 \mathrm{WE}$ als latente Wärme, also im Ganzen 93 WE/kg erforderlich sein. Mit 2000 WE im Grenzfall würden rund $21 \mathrm{~kg}$ Eis geschmolzen werden können.

Durch die Untersuchung zweier Grenzfälle, zwischen denen der tatsächlich im Inlandeis eintretende Vorgang liegen muß, nämlich der Erzeugung von Schmelzwasser ausschließlich am Grunde in einer Menge, die zwischen 0 und $21 \mathrm{~kg}$ je qm liegen muß, ist schon ein engerer Rahmen gegeben, der durch begründete Schätzung noch enger gezogen werden kann.

Die tatsächlichen Vorgänge. Um diesen näher zu kommen, muß die oben zugrunde gelegte schematische Betrachtungsweise der Wirklichkeit besser angepaßt werden. Das angenommene senkrechte Absinken der Eisschichten wird zwar im Gebiet der Eisscheide annähernd zutreffend sein. Der auf Eismitte von der deutschen Grön- 
landexpedition ausgehobene Schacht von $15 \mathrm{~m}$ Tiefe ist in Jahresfrist nicht durch horizontale Schubkräfte zerstört worden (K. WEGENER, III, S. 203). Solche seitlichen Schubkräfte sind eben auf der Eisscheide in der Nähe der Oberfläche überhaupt nicht vorhanden. In tieferen Schichten und zunehmend mit der Entfernung von der Eisscheide dagegen wird allmählich eine Horizontalbewegung einsetzen: die absinkenden Firnmassen schwenken von der senkrechten in eine geneigte Richtung ab, wobei dann auch die oberen Schichten passiv der seitlichen Bewegung der unteren folgen.

Unter solchen Umständen stellen sich folgende Änderungen gegenüber der obigen schematischen Annahme ein:

i. Durch die Richtungsänderung der Eismassen wird die innere Reibung und Wärmeerzeugung gegenüber den Verhältnissen bei ungestörtem, senkrechten Absinken erhöht.

2. Aber durch den allmählichen Übergang der Bewegung in eine annähernd horizontale wird ein Teil des Eises, der schon um einiges erwärmt worden ist, aus dem Zentralgebiet in die Randzone befördert, so daß ein erheblicher Teil der schon erzeugten Wärme dem Wärmehaushalt des Zentralgebiets verloren geht.

3. Auch von dem Vorrat an potentieller Energie, der im Zentralgebiet zur Wärmeerzeugung noch nicht verbrauche war, geht aus dem gleichen Grunde ein großer Teil in die Randzone über und kommt erst hier zur Wirkung. Nur ein kleiner Teil des Eises gelangt im Zentralgebiet in tiefere Schichten; entsprechend vermindert sich die hier noch wirksam werdende potentielle Energie.

Zu Punkt 1 ist zusätzlich noch folgendes zu erwähnen: Ungestörtes Absinken des E1ses, ohne daß ein Anlaß zu Reibungsarbeit gegeben ist, führt noch zu keiner Wärmeerzeugung. Der Druck summiert sich von oben nach unten; er überträgt sich über die Eisschichten bis an die Stellen, wo Reibungsarbeit geleistet wird, und wird auf seinem Wege nur vermindert durch Arbeitsleistung infolge innerer Eisreibung.

Nur unmittelbar da, wo Reibung a f tritt, entsteht Wärme, also an Stellen der Zusammenpressung oder Richtungsänderung des Eises oder am festen Boden und seinen Aufragungen. Daraus geht hervor, daß e in e gle ichmäßige thermische Tiefenstufe im Inlandeis nicht bestehen kann. Es sind Berechnungen der Tiefe, in welcher der Schmelzpunkt im Inlandeis erreicht wird, vorgenommen worden, ausgehend von der Differenz der Temperaturen an der Oberfläche und in einer gewissen geringen Tiefe. Soweit sie eine gleichmäßige thermische Tiefenstufe voraussetzen, können sie schon aus diesem Grunde nicht zu einem richtigen Ergebnis führen.

Aus den obigen Úberlegungen unter 1 bis 3 geht hervor, daß die wahrscheinlich weit überwiegende Menge der schon erzeugten Wärme und der restlichen potentiellen Energie dem Wärmehaushalt des Zentralgebiets verloren geht.

Zweifellos wird an der Sohle durch die Reibung des Eises am Untergrund Wärme erzeugt. Indessen ist die Eisgeschwindigkeit im Zentralgebiet überhaupt gering und ferner an der Sohle selbst am kleinsten; das Höchstmaß wird erst in einem etwas höheren Niveau erreicht. Wenn schon oben für den zweiten Grenzfall eine Maximalmenge geschmolzenen Eises von jährlich nur $21 \mathrm{~kg} / \mathrm{qm}$, entsprechend einer Wasserschicht von rund $2 \mathrm{~cm} / \mathrm{Jahr}$, errechnet wurde, so kann hiervon unter den geschilderten Umständen nur ein kleiner Bruchteil tatsächlich zum Schmelzen kommen.

$\mathrm{Zu}$ diesem Teil tritt noch die Schmelzwassermenge hinzu, die durch die Wirkung des aus der Erde kommenden Wärmestromes gebildet wird. Sie ist von B. Brockamp auf eine jährliche Wasserschicht von 0,5 bis $1 \mathrm{~cm}$ berechnet (K. WEGENER, III, S. 50). Beide Mengen zusammen werden wahrscheinlich die jährliche Höhe von $1 \mathrm{~cm}$ nicht wesentlich überschreiten. 
Diese geringe Menge hat sich allerdings noch nicht auf seismischem Wege nachweisen lassen (ebenda S. 37). Das ist verständlich, weil bei gleichmäßigem Abfluß der geringen Jahresmenge jeweils nur eine minimale Schicht vorhanden ist. Indessen wird auf Seite 27 im Bilde der Gletscherbruch des Kamarujuk-Gletschers gezeigt, in dem ein auch im Winter fließender Gletscherbach sichtbar ist, wodurch ein ständiger subglaziärer Schmelzvorgang bewiesen wird.

$\mathrm{Zu}$ den geschilderten Vorgängen kommen im Randgebiet noch die vielseitige $\mathrm{Ab}$ lation sowie die Kalbung hinzu. Hier sollten jedoch nur die Verhältnisse des zentralen Bereichs behandelt werden.

$\mathrm{Z}$ us a mmenf assung. Aus den Darlegungen und Berechnungen geht Folgendes f ür das Z entralgebiet des Inlandeises hervor:

1. Der Schmelzpunkt wird im Innern des Eises überhaupt nicht erreicht.

2. Die Schmelzwassermenge an der Sohle kann zusammen mit dem vom Erdwärmestrom erzeugten Schmelzwasser nur sehr geringfügig sein.

Schriftenverzeichnis

Brinkmann, R.: Kaysers Abriß der Geologie I, 6. Aufl. 1940.

KANNENBerg, H.: Erdkunde 5, S. 329. Bonn 1951.

WegenER, K.: Wissenschaftliche Ergebnisse der Deutschen Grönlandexpedition Alfred Wegener. 1933-1940.

Woldstedt, P.: „Der Bewegungsvorgang beim Inlandeis“. - Peterm. Mitt. 1952, Heft 4.

Manuskr. eingeg. 22. 11. 1954.

Anschrift des Verf.: Reg.-Dir. a. D. Paul Jaspersen, (24b) Kiel, Goethestraße 8. 